\title{
Prevention, monitoring and treatment of cardiovascular adverse events in myeloma patients receiving carfilzomib A consensus paper by the European Myeloma Network and the Italian Society of Arterial Hypertension
}

\author{
- S. Bringhen ${ }^{1}$ (D) A. Milan² ${ }^{2}$ M. D’Agostino ${ }^{1}$, C. Ferri ${ }^{3}$, R. Wäsch ${ }^{4}$, F. Gay ${ }^{1}$, A. Larocca ${ }^{1}$, M. Offidani ${ }^{5}$, \\ S. Zweegman ${ }^{6}$, E. Terpos $^{7}$, H. Goldschmidt ${ }^{8}$, M. Cavo ${ }^{9}$, H. Ludwig ${ }^{10}$, C. Driessen ${ }^{11}$, H. W. Auner ${ }^{12}$, J. Caers ${ }^{13}$, \\ M. Gramatzki ${ }^{14}$, M. A. Dimopoulos ${ }^{7}$, M. Boccadoro ${ }^{1}$, H. Einsele ${ }^{15}$, P. Sonneveld ${ }^{16}$ \& M. Engelhardt ${ }^{4}$ \\ From the ${ }^{1}$ Myeloma Unit, Division of Hematology, University of Torino, Azienda Ospedaliero-Universitaria Città della Salute e della Scienza di \\ Torino; ${ }^{2}$ Department of Medical Sciences, Internal Medicine and Hypertension Division, AOU Città della Salute e della Scienza di Torino, Rete \\ Oncologica Piemontese, University of Torino, Torino, Italy; ${ }^{3}$ University of L'Aquila, MeSVA Department - San Salvatore Hospital, Division of \\ Internal Medicine \& Nephrology, Coppito, Italy; ${ }^{4}$ Department of Medicine I, Hematology, Oncology \& Stem Cell Transplantation, Medical \\ Center - University of Freiburg, Faculty of Medicine, University of Freiburg, Freiburg, Germany; ${ }^{5}$ Clinica di Ematologia, AOU Ospedali Riuniti \\ di Ancona, Ancona, Italy; ${ }^{6}$ Amsterdam UMC, Vrije Universiteit Amsterdam, VU University Medical Center, Department of Hematology, Cancer \\ Center Amsterdam, Amsterdam, the Netherlands; ${ }^{7}$ Department of Clinical Therapeutics, National and Kapodistrian University of Athens, \\ School of Medicine, Athens, Greece; ${ }^{8}$ University Clinic Heidelberg, Internal Medicine V and National Center for Tumor Diseases (NCT), \\ Heidelberg, Germany; 'Seràgnoli' Institute of Hematology and Medical Oncology, University of Bologna, Bologna, Italy; ${ }^{10} 1$. Medical \\ Department and Oncology, Wilhelminenspital Wien, Vienna, Austria; ${ }^{11}$ Department of Oncology and Hematology, Kantonsspital St. Gallen, \\ St. Gallen, Switzerland; ${ }^{12}$ Faculty of Medicine, Department of Medicine, Imperial College London, London, UK; ${ }^{13}$ Department of Hematology, \\ Domaine University Liege, Liege, Belgium; ${ }^{14}$ Division of Stem Cell Transplantation and Immunotherapy, University of Kiel, Kiel; ${ }^{15}$ Department \\ of Internal Medicine II, University Hospital Würzburg, Würzburg, Germany; and ${ }^{16}$ Department of Hematology, Erasmus Medical Center, \\ Rotterdam, the Netherlands
}

Abstract. Bringhen S, Milan A, D’Agostino M, Ferri C, Wäsch R, Gay F, Larocca A, Offidani M, Zweegman $\mathrm{S}$, Terpos E, Goldschmidt H, Cavo M, Ludwig H, Driessen C, Auner HW, Caers J, Gramatzki M, Dimopoulos MA, Boccadoro M, Einsele $H$, Sonneveld P, Engelhardt M (University of Torino, Torino, Italy; Rete Oncologica Piemontese, Torino, Italy; University of L'Aquila, Coppito, Italy; University of Freiburg, Freiburg, Germany; AOU Ospedali Riuniti di Ancona, Ancona, Italy; Vrije Universiteit Amsterdam, Amsterdam, the Netherlands; National and Kapodistrian University of Athens, Athens, Greece; University Clinic Heidelberg, Heidelberg, Germany; University of Bologna, Bologna, Italy; Wilhelminenspital Wien, Vienna, Austria; Kantonsspital St. Gallen, St. Gallen, Switzerland; Imperial College London, London, UK; Domaine University Liege, Liege, Belgium; University of Kiel, Kiel, Germany University Hospital Würzburg, Würzburg, Germany; and Erasmus Medical Center, Rotterdam, the Netherlands). Prevention, monitoring and treatment of cardiovascular adverse events in myeloma patients receiving carfilzomib A consensus paper by the European Myeloma Network and the Italian Society of Arterial Hypertension. J Intern Med 2019; 286: 63-74.
Background. The novel proteasome inhibitor carfilzomib alone or in combination with other agents is already one of the standard therapies for relapsed and/or refractory multiple myeloma (MM) patients and produces impressive response rates in newly diagnosed MM as well. However, carfilzomibrelated cardiovascular adverse events (CVAEs) including hypertension (all grades: 12.2\%; grade $\geq 3$ : $4.3 \%$ ), heart failure (all grades: $4.1 \%$; grade $\geq 3$ : $2.5 \%$ ) and ischemic heart disease (all grades: 1.8\%; grade $\geq 3: 0.8 \%$ ) - may lead to treatment suspensions. At present, there are neither prospective studies nor expert consensus on the prevention, monitoring and treatment of CVAEs in myeloma patients treated with carfilzomib.

Methods. An expert panel of the European Myeloma Network in collaboration with the Italian Society of Arterial Hypertension and with the endorsement of the European Hematology Association aimed to provide recommendations to support health professionals in selecting the best management strategies for patients, considering the impact on outcome and the risk-benefit ratio of diagnostic and therapeutic tools, thereby achieving myeloma response with novel combination approaches whilst preventing CVAEs. 
Results. Patients scheduled to receive carfilzomib need a careful cardiovascular evaluation before treatment and an accurate follow-up during treatment.

Conclusions. A detailed clinical assessment before starting carfilzomib treatment is essential to identify patients at risk for CVAEs, and accurate monitoring of blood pressure and of early signs and symptoms suggestive of cardiac dysfunction remains pivotal to safely administer carfilzomib without treatment interruptions or dose reductions.

Keywords: adverse events, blood pressure monitoring, cardiovascular toxicity, carfilzomib, clinical assessment, multiple myeloma.

\section{Introduction}

Carfilzomib (CFZ), a second-generation proteasome inhibitor (PI), is active as a single agent and in combination with other anti-multiple myeloma (MM) agents. CFZ has been approved in Europe for the treatment of relapsed/refractory MM (RRMM), in combination with lenalidomide and/ or dexamethasone, based on the randomized trials ASPIRE [1] and ENDEAVOR [2]. In ASPIRE, 792 patients were randomized to receive $\mathrm{CFZ}$ with lenalidomide and dexamethasone (KRd) or lenalidomide and dexamethasone alone (Rd). In ENDEAVOR, 929 patients were randomized to receive $\mathrm{CFZ}$ with dexamethasone $(\mathrm{Kd})$ or bortezomib with dexamethasone (Vd). CFZ-based regimens, KRd and $\mathrm{Kd}$, are the first therapy combinations to demonstrate a significant overall survival (OS) advantage $(21 \%$ reduction of risk of death, resulting in nearly 8 additional months of OS) for relapsed MM patients versus recent standards of care (Rd and Vd) [3].

However, in ASPIRE and ENDEAVOR, KRd and Kd treatments were associated with higher than expected rates of hypertension (all grade: 14.3\% and $16 \%$; grade $\geq 3: 4.3 \%$ and $9 \%$, heart failure (HF; all grades: $6.4 \%$ and $3 \%$; grade $\geq 3: 3.8 \%$ and $4.8 \%$ ) and ischaemic heart disease (all grades: $5.9 \%$ and $0.9 \%$; grade $\geq 3: 3.3 \%$ and $1.7 \%$, respectively) $[1,2]$.

A recent meta-analysis performed on 24 clinical studies with available nonhaematological adverse events data associated with CFZ treatment showed that the incidence of all-grade and grade $\geq 3$ cardiovascular adverse events (CVAEs) was $18.1 \%$ and $8.2 \%$, respectively. In randomized clinical trials, the relative risk (CFZ vs controls) of allgrade and grade $\geq 3$ CVAEs were 1.8 and 2.2, respectively [4]. The most frequent CVAEs during treatment with $\mathrm{CFZ}$ are hypertension (all grades: $12.2 \%$; grade $\geq 3: 4.3 \%$ ), heart failure (all grades:
4.1\%; grade $\geq 3: 2.5 \%$ ) and ischaemic heart disease (all grades: $1.8 \%$; grades $\geq 3$ : $0.8 \%$ ). Dyspnoea is another particular issue, which is reported in patients treated with $\mathrm{CFZ}$ with an incidence of $23.9 \%$ (all grades) and $3.2 \%$ (grades $\geq 3$ ). Although the causes are not clear, it could be a symptom related to cardiovascular conditions, such as cardiac failure, as well as pulmonary complications. Venous thromboembolic events and thrombotic microangiopathy have also been reported in patients who received CFZ [1-11].

The mechanisms by which CFZ induces CVAEs are poorly understood. However, speculations can be made on its irreversible and highly potent proteasome inhibition activity that could differentiate its safety profile from that of bortezomib [12]. Cardiac stress produces misfolded proteins; proteasomemediated degradation of these toxic products is pivotal to preserve cellular function in some patients [13, 14]. Moreover, the levels of nitric oxide, an important mediator of endothelial function, could also be modulated by proteasome activity, and decreased nitric oxide levels could impair vasodilatation, inducing hypertension and cardiac dysfunction [15].

Neither prospective studies nor expert consensus have been reported so far on the prevention, monitoring and treatment of CVAEs in MM patients treated with CFZ. Therefore, this European Myeloma Network (EMN) consensus paper, in collaboration with the Italian Society of Arterial Hypertension (SIIA) and the support of the European Hematology Association (EHA), aims to help physicians to prevent and manage CVAEs during CFZ treatment, thereby improving the risk/benefit ratio of this widely used drug.

The currently available guidelines on cancer treatments and cardiotoxicity are based on studies performed on tumour entities other than MM and are discussed in the Supplementary Appendix. 
Table 1 Factors influencing the stratification of total cardiovascular risk [36]

Demographic characteristics and laboratory parameters

$\operatorname{Sex}^{\mathrm{a}}$ (men > women)

Age $^{\mathrm{a}}$

Smoking (current or past history) ${ }^{\mathrm{a}}$

Total cholesterol ${ }^{\mathrm{a}}$ and HDL-C

Uric acid

Diabetes $^{a}$

Overweight or obesity

Family history of premature CVD (men aged $<55$ years and women aged $<65$ years)

Family or parental history of early-onset hypertension

Early-onset menopause

Sedentary lifestyle

Psychosocial and socio-economic factors

Heart rate (resting values $>80$ beats $\min ^{-1}$ )

Asymptomatic HMOD

Arterial stiffening:

Pulse pressure (in older people) $\geq 60 \mathrm{mmHg}$

Carotid-femoral PWV $>10 \mathrm{~m} \mathrm{~s}^{-1}$

ECG LVH (Sokolow-Lyon index $>35 \mathrm{~mm}$, or $\mathrm{R}$ in $\mathrm{aVL} \geq 11 \mathrm{~mm}$; Cornell voltage duration product $>2440 \mathrm{~mm}$. $\mathrm{ms}$, or Cornell voltage $>28 \mathrm{~mm}$ in men or $>20 \mathrm{~mm}$ in women)

Echocardiographic LVH [LV mass index: men $>50 \mathrm{~g} / \mathrm{m}^{2.7}$; women $>47 \mathrm{~g} / \mathrm{m}^{2.7}$ (height in $\mathrm{m}^{2.7}$ ); indexation for BSA may be used in normal-weight patients; LV mass/BSA g/ $\mathrm{m}^{2}>115$ (men) and $>95$ (women)]

Microalbuminuria (30-300 mg/24 h), or elevated albumin-creatinine ratio (30-300 mg/g; 3.4-34 mg mmol ${ }^{-1}$;

preferentially on morning spot urine) $)^{\mathrm{b}}$

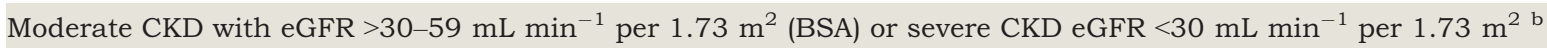

Ankle-brachial index $<0.9$

Advanced retinopathy: haemorrhages or exudates, papilloedema

Established $C V$ or renal disease

Cerebrovascular disease: ischaemic stroke, cerebral haemorrhage, TIA

CAD: myocardial infarction, angina, myocardial revascularization

Presence of atheromatous plaque on imaging

Heart failure, including HFpEF

Peripheral artery disease

Atrial fibrillation

BSA, body surface area; CAD, coronary artery disease; CKD, chronic kidney disease; CV, cardiovascular; CVD, cardiovascular disease; ECG, electrocardiogram; eGFR, estimated glomerular filtration rate; HDL-C, HDL cholesterol; $\mathrm{HFpEF}$, heart failure with preserved ejection fraction; HMOD, hypertension-mediated organ damage; LV, left ventricular; LVH, left ventricular hypertrophy; PWV, pulse wave velocity; SCORE, Systematic COronary Risk Evaluation; TIA, transient ischaemic attack.

${ }^{\mathrm{a}} \mathrm{CV}$ risk factors included in the SCORE system.

${ }^{\mathrm{b}}$ Proteinuria and reduced eGFR are independent risk factors.

Source: Bryan Williams et al. 2018 ESC/ESH Guidelines for the management of arterial hypertension. European Heart Journal (2018); 39 (33): 3021-104; Table 4, p. 3031, https:/ / doi.org/ 10.1093/eurheartj/ehy339. Reproduced by permission of Oxford University Press on behalf of the European Society of Cardiology. All rights reserved. CEuropean Society of Cardiology and European Society of Hypertension 2018. For permissions, please email journals.permissions@oup.com. Please visit: https://www.escardio.org/Guidelines/Clinical-Practice-Guidelines/Arterial-Hypertension-Management-of. 


\begin{tabular}{|c|c|c|c|c|c|}
\hline \multirow[b]{2}{*}{$\begin{array}{l}\text { Hypertension } \\
\text { disease } \\
\text { staging }\end{array}$} & \multirow[b]{2}{*}{$\begin{array}{l}\text { Other risk factors, } \\
\text { HMOD or disease }\end{array}$} & \multicolumn{4}{|c|}{$\mathrm{BP}(\mathrm{mmHg})$ grading } \\
\hline & & $\begin{array}{l}\text { High normal } \\
\text { SBP 130-139 } \\
\text { DBP 85-89 }\end{array}$ & $\begin{array}{l}\text { Grade } 1 \\
\text { SBP 140-159 } \\
\text { DBP 90-99 }\end{array}$ & $\begin{array}{c}\text { Grade } 2 \\
\text { SBP 160-179 } \\
\text { DBP 100-109 }\end{array}$ & $\begin{array}{c}\text { Grade } 3 \\
\mathrm{SBP} \geq 180 \\
\text { or } \mathrm{DBP} \geq 110\end{array}$ \\
\hline \multirow{3}{*}{$\begin{array}{c}\text { Stage } 1 \\
\text { (uncomplicated) }\end{array}$} & $\begin{array}{l}\text { No other risk } \\
\text { factors }\end{array}$ & Low risk & Low risk & Moderate risk & High risk \\
\hline & 1 or 2 risk factors & Low risk & Moderate risk & $\begin{array}{l}\text { Moderate to } \\
\text { high risk }\end{array}$ & High risk \\
\hline & $\geq 3$ risk factors & $\begin{array}{l}\text { Low to } \\
\text { Moderate risk }\end{array}$ & $\begin{array}{l}\text { Moderate to } \\
\text { high risk }\end{array}$ & High Risk & High risk \\
\hline $\begin{array}{c}\text { Stage } 2 \\
\text { (asymptomatic } \\
\text { disease) }\end{array}$ & $\begin{array}{c}\text { HMOD, CKD grade } \\
3 \text { or diabetes } \\
\text { mellitus without } \\
\text { organ damage }\end{array}$ & $\begin{array}{l}\text { Moderate to } \\
\text { high risk }\end{array}$ & High risk & High risk & $\begin{array}{l}\text { High to } \\
\text { very high risk }\end{array}$ \\
\hline $\begin{array}{c}\text { Stage } 3 \\
\text { (established } \\
\text { disease) }\end{array}$ & $\begin{array}{l}\text { Established CVD, } \\
\text { CKD grade } \geq 4 \text { or } \\
\text { diabetes mellitus } \\
\text { with organ damage }\end{array}$ & Very high risk & Very high risk & Very high risk & Very high risk \\
\hline
\end{tabular}

Fig. 1 Classification of hypertension stages according to blood pressure levels, presence of cardiovascular risk factors, hypertension-mediated organ damage or comorbidities defined by the 2018 ESC/ESH Guidelines. CV risk is illustrated for a middle-aged male. The CV risk does not necessarily correspond to the actual risk at different ages. The use of the SCORE system is recommended for formal estimation of $C V$ risk for treatment decisions. BP, blood pressure; CKD, chronic kidney disease; CV, cardiovascular; DBP, diastolic blood pressure; HMOD, hypertension-mediated organ damage; SBP, systolic blood pressure; SCORE, Systematic COronary Risk Evaluation. Source: Bryan Williams et al. 2018 ESC/ESH Guidelines for the management of arterial hypertension. European Heart Journal (2018); 39 (33): 3021-104; Fig. 1, p. 3034, https://doi. org/ 10.1093/eurheartj/ehy339. Reproduced by permission of Oxford University Press on behalf of the European Society of Cardiology. All rights reserved. (C) European Society of Cardiology and European Society of Hypertension 2018. For permissions, please email journals.permissions@oup.com. Please visit: https://www.escardio.org/Guidelines/ClinicalPractice-Guidelines/Arterial-Hypertension-Management-of.

\section{Risk factors}

To identify patients at increased risk for CVAEs, the first step is a careful baseline assessment of cardiovascular risk factors and prior cardiovascular diseases (Table 1, Fig. 1) as well as prior exposition to cardiotoxic treatments (e.g. anthracyclines or chest radiotherapy; Table S2) [5]. No data are available on prior exposition to proteasome inhibitors as a risk factor.

The most frequent CVAE is hypertension, which is itself a trigger event for other CVAEs, such as heart failure and ischaemic heart disease. According to the ESH/ESC guidelines for the management of arterial hypertension [16], the estimation of all cardiovascular risks should be performed with a detailed stratification (Table 1, Fig. 1) or with the handier Systematic COronary Risk Evaluation
(SCORE) model that estimates the risk of dying from cardiovascular disease over 10 years [17].

Arterial hypertension is defined by a systolic blood pressure $\geq 140 \mathrm{mmHg}$ and/or a diastolic blood pressure $\geq 90 \mathrm{mmHg}$. Methods used to monitor patients' blood pressure are as follows:

1 Office blood pressure monitoring: this is usually higher than out-of-office blood pressure and home blood pressure and the difference increases as office blood pressure increases.

2 Out-of-office blood pressure monitoring: it provides many blood pressure measurements away from the medical environment, which correlates better with actual blood pressure than office blood pressure. Outof-office blood pressure is commonly assessed by 
Table 2 Main features of ambulatory blood pressure monitoring and home blood pressure monitoring

\begin{tabular}{lll} 
& ABPM & HBPM \\
\hline Brief & Blood pressure & Blood pressure \\
description & measurement & self-measurements \\
& with a portable & daily for at least \\
& blood pressure & 3-4 days and \\
& measuring & preferably for 7 \\
& device for a & consecutive days \\
& 24 h period & \\
Primary care & - & + \\
Specialist care & + & - \\
Cheap & - & ++ \\
24 h & ++ & - \\
Daily activity & ++ & - \\
Sleep & ++ & - \\
Long period & - & \\
(at least & & \\
7 days) & &
\end{tabular}

ABPM, ambulatory blood pressure monitoring; HBPM, home blood pressure monitoring.

ambulatory blood pressure monitoring (ABPM) or home blood pressure monitoring (HBPM; Table 2).

For initial assessment, HBPM may be better suitable in primary care and ABPM in specialist care. However, borderline or abnormal findings on HBPM should be confirmed with ABPM [18], which is currently considered the reference for out-ofoffice blood pressure. Furthermore, defining values for arterial hypertension according to HBPM (systolic blood pressure $>135 \mathrm{mmHg}$ and/or a diastolic blood pressure $>85 \mathrm{mmHg}$ ) are slightly lower than the classical definition of arterial hypertension [16]. Patients should be routinely trained for selfmeasurement of blood pressure to optimize followup, for which HBPM is more suitable than ABPM.

Hypertension-mediated organ damage (HMOD; Figure S1) predicts cardiovascular death independently of ESH/ESC guidelines and/or SCORE model (http://www.heartscore.org), so it should be accurately screened.

\section{Role of biomarkers}

Cardiac biomarkers are not essential parameters in clinical practice for the early detection of cardiotoxicity, even though they have a role in patients who develop cardiotoxicity. Available data on cardiac biomarkers are described in the Supplementary Appendix.

\section{Role of imaging}

Evaluation of cardiac organ damage represents a pivotal step in cardiovascular risk stratification of the general population [19]. A similar approach to risk stratification could be applied to MM patients candidates for CFZ therapy. A comprehensive assessment requires evaluation of both structural and functional features, with different diagnostic tools.

\section{Standard echocardiography}

The most frequently used parameter for routine cardiotoxicity monitoring is LVEF. A LVEF of $>52 \%$ for men and $>54 \%$ for women is considered normal [20, 21]. A LVEF drop of $>10 \%$ or $>5 \%$ with heart failure symptoms is considered diagnostic of cardiotoxicity. LVEF before chemotherapy is considered as a predictor of subsequent cardiotoxicity. Nevertheless, the prognostic value and the timing of serial measurements of LVEF during treatment for cardiotoxicity detection and monitoring are still controversial. A sub-analysis of the ENDEAVOR study on 151 patients randomized between Kd vs $\mathrm{Vd}$ failed to demonstrate a lower LVEF in Kdtreated patients and serial screening with echocardiography in unselected patients was not helpful to mitigate the risk of CVAEs [7]. However, echocardiography assessment can be helpful to obtain a baseline evaluation of LVEF in patients before treatment and in case of established CVAEs for diagnostic purposes.

\section{Advanced echocardiographic evaluation}

Myocardial deformations can be studied using different ultrasound techniques as Tissue Doppler and $2 \mathrm{D}$ and $3 \mathrm{D}$ speckle tracking echocardiography [22]. Tissue Doppler is more sensitive than LVEF assessments in recognizing chemo- and/or radiotherapy-induced left ventricle systolic dysfunction, early cardiotoxicity even for low-dose chemotherapy and differences in regional myocardial function secondary to localized drug damage (i.e. the interventricular septum) [23]. However, Tissue Doppler has several limitations, such as a low reproducibility with angle dependency, a limited spatial resolution, a high sensitivity to signal noise and a high inter-observer variability. 
Global longitudinal strain (GLS) assessed using automated speckle tracking echocardiography is an emerging technique for detecting and quantifying subtle disturbances in LV systolic function. GLS reflects the longitudinal contraction of the myocardium and its accuracy has been validated against tagged magnetic resonance imaging [24]. GLS provides more consistent results than radial and circumferential myocardial deformation analyses in the early recognition of myocardial damage, the prediction of late cardiotoxicity onset and the planning of cardio-protection strategies. There is evidence that GLS is the most sensitive and specific measurement for the early detection of subclinical myocardial injury [25]. The American Society of Echocardiography (ASE) and European Association of Cardiovascular Imaging (EACVI) consensus [26] suggested a practical approach for GLS use in patients undergoing chemotherapy. More precisely, a GLS reduction $<8 \%$ from baseline is not meaningful, but $>15 \%$ from baseline is very likely abnormal.

In our experience, 28 consecutive RRMM patients treated with bortezomib (BTZ) and CFZ were compared with a population of 22 non-MM control subjects, matched for age, sex and mean $24 \mathrm{~h}$ blood pressure [27]. All patients underwent trans-thoracic echocardiography, ABPM and a pulse wave velocity study to assess cardiac morphology and function, blood pressure load and arterial stiffness. Pulse wave velocity was similar between PI-treated patients and controls. GLS was the only echocardiographic parameter significantly decreased in PItreated $(P=0.02)$ and in CFZ-treated patients $(P=0.002)$, even after correction for the main cardiac function parameters $(P=0.01$ and $P=0.036$, respectively). Amongst $\mathrm{CFZ}$ patients, we also found increased values of LV mass indexed by body surface area $(P=0.047)$. Moreover, in this cohort, the cumulative dose of $\mathrm{CFZ}$ was associated with a more prominent modification of GLS and LV mass indexed by body surface area [27].

\section{Cardiac magnetic resonance}

Cardiac magnetic resonance is the reference standard in assessing LV and right ventricle volumes and function, and it is now extensively used to detect acute and chronic cardiac chemotherapy complications [28]. Cardiac magnetic resonance is superior to echocardiography for many reasons, but it has several limitations (low availability, high costs, contraindication to ferromagnetic devices).
Table 3 The role of imaging in the management of CVAEs

Echocardiography is the first-choice method for evaluating patients before, during and after chemotherapy, and Left Ventricular Ejection Fraction (LVEF) is the primary technique Cardiotoxicity cannot be predicted by LVEF alone but an accurate echocardiographic investigation is strongly recommended, if available, to integrate the standard examination with data from different imaging techniques (Tissue Doppler Imaging and speckle tracking echocardiography)

Diastolic indices are not useful for early detection of cardiotoxicity because of their inability to predict heart failure

Global Longitudinal Strain (GLS) should be performed only by speckle tracking echocardiography for a sensitive diagnosis of chemotherapy-induced cardiac damage, and the same ultrasound equipment should be used for serial examinations

Cardiac magnetic resonance is recommended for LVEF quantification when the quality of echocardiogram is suboptimal. Furthermore, cardiac magnetic resonance is suggested for confirming a LVEF $<53 \%$

Multigated angiography provides a highly reproducible quantification of LVEF during cancer therapy, but radiation exposure remains its main limitation. Therefore, this technique should be considered only when first-line echocardiography and second-line cardiac magnetic resonance are unavailable

\section{Radionuclide angiography (multigated angiography-MUGA)}

Multigated angiography has been the 'gold standard' imaging technique to evaluate LV systolic function in patients undergoing chemotherapy for many years [29]. The main limit of MUGA is radiation exposure, which reduces its use after increasing availability of other imaging techniques. Multigated angiography also does not provide comprehensive information on right ventricle function, left and right atrial size, and presence or absence of valvular or pericardial disease. The ASE and EACVI positions on the role of imaging techniques in cardiotoxicity management are summarized in Table 3 [26]. 
Prevention of chemotherapy-induced cardiotoxicity in cancer patients

\section{$\beta$-blockers}

It is well known that chronic activation of the sympathetic nervous system plays an important role in heart failure pathogenesis; therefore, $ß-$ blockers should be used in all patients with reduced LVEF to prevent heart failure-related hospitalization and mortality. However, the use of $ß$ blockers in oncologic patients undergoing chemotherapy, with asymptomatic LV dysfunction, is as yet not well established [30].

\section{Renin-angiotensin-aldosterone system: angiotensin-converting} enzyme inhibitors (ACEi) and angiotensin II receptor blockers (ARB)

The activation of renin-angiotensin-aldosterone system is one of the potential mechanisms involved in chemotherapy-induced cardiotoxicity. Angiotensin-converting enzyme inhibitors attenuate oxidative stress, reduce interstitial fibrosis, and improve intracellular calcium handling, cardiomyocyte metabolism and mitochondrial function, and there is good evidence on their efficacy in anthracycline-induced cardiotoxicity [30, 31]. The efficacy of a combined therapy with ACEi and $\$-$ blockers in preventing cardiotoxicity has been demonstrated in the OVERCOME trial [32]. This study evaluated the efficacy of enalapril and carvedilol in preventing chemotherapy-induced LV systolic dysfunction in patients with haematologic diseases treated with conventional chemotherapy. Results showed a lower reduction in $\mathrm{LV}$ function and a lower incidence of heart failure in patients treated with this combination treatment compared to placebo. As a consequence, ACEi and 3 -blockers proved beneficial in conventional chemotherapy-induced cardiotoxicity. The PRADA trial demonstrated that in patients treated for early breast cancer with anthracycline-containing regimens with or without trastuzumab and radiation, the treatment with the ARB candesartan provided protection against early decline in global left ventricular function, whilst no short-term beneficial effect was observed for the $\beta$-blocker metoprolol alone [33].

\section{Nutritional supplementation and exercise training}

Nonpharmacologic strategies to reduce chemotherapy-induced cardiotoxicity are discussed in the Supplementary Appendix.
Suggestions for workup in MM patients candidate to CFZ therapy

In general, no differences in CVAEs were observed between RRMM and newly diagnosed MM patients, nor amongst different treatment combinations [4]. According to these data, an appropriate cardiovascular risk assessment is recommended for all patients receiving CFZ (Figs 1 and 2):

- Patients with no cardiovascular risks and normal blood pressure can start treatment with $\mathrm{CFZ}$ immediately.

- Patients with low-moderate risk should correct modifiable risk factors and hypertension.

- High-risk patients should undergo a case by case evaluation considering the risk/benefit.

- Very high-risk patients are more likely to have non-modifiable risk factors; treatment options other than CFZ should be considered.

A slightly higher incidence of CVAEs was reported in patients receiving higher doses of CFZ $\left(\geq 45 \mathrm{mg} \mathrm{m}^{-2}\right)$ [4] and in elderly patients ( $\geq 75$ years): thus, these subgroups should be strictly monitored. No suggestions could be done for the use of CFZ in amyloidosis patients outside of clinical trials because it is not approved by the US Food and Drug Administration (FDA) or the European Medicines Agency (EMA) and few data are available.

\section{Before starting therapy}

Clinicians should perform a comprehensive workup:

- Medical history: to determine previous cardiovascular events and risk factors (hypertension, diabetes, dyslipidemia, obesity, smoking, etc.) as well as prior exposition to cardiotoxic cancer treatments (Table S2);

- Physical examination:

- Blood pressure (hypertension is a potent and modifiable risk factor for cardiac dysfunction onset and should be assessed before starting treatment);

- Heart auscultation to identify murmurs (significant valvular heart disease is a risk factor for cardiac dysfunction); 


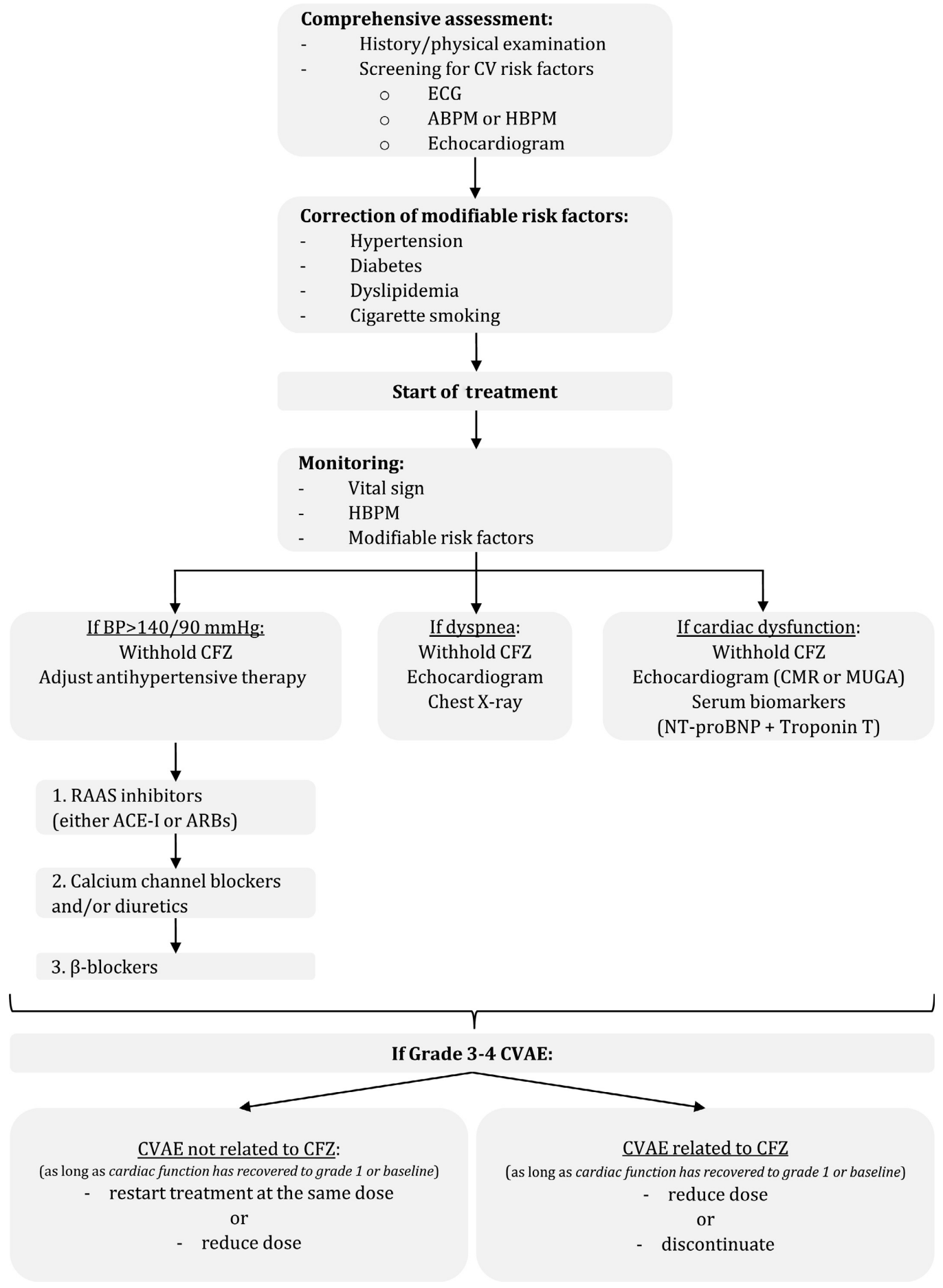

Fig. 2 Flowchart for patient selection and evaluation before and during treatment with CFZ. CV, cardiovascular; ECG, electrocardiogram; ABPM, ambulatory blood pressure monitoring; HBPM, home blood pressure monitoring; BP, blood pressure; CFZ, carfilzomib; CMR, cardiac magnetic resonance; MUGA, multigated angiography; RAAS, renin-angiotensinaldosterone system; ACEi, angiotensin-converting enzyme inhibitors; ARB, angiotensin II receptor blockers. 
- Signs of heart failure (elevated venous pressure, lung crackles or pedal oedema);

- 12-lead electrocardiogram (ECG) to detect possible markers of structural heart disease, including LV damage/dysfunction, arrhythmias (atrial fibrillation, atrial flutter, heart block), evidence of previous myocardial infarction (Q-waves, left bundle branch block) and evidence of LV hypertrophy;

- LVEF measurement using echocardiography, cardiac magnetic resonance or MUGA to assess asymptomatic cardiac organ damage and to have a baseline evaluation useful as a reference in case of CVAEs;

- ABPM/HBPM (Table 2) to detect unknown, borderline or uncontrolled hypertension.

Patients with home blood pressure >135/ $85 \mathrm{mmHg}$ should be treated; those already receiving hypertensive medication may need adjustments in their medication to manage their blood pressure before the start of $\mathrm{CFZ}$ treatment.

\section{During therapy}

- Clinicians should manage modifiable cardiovascular risk factors (smoking, hypertension, diabetes, dyslipidemia, obesity) in all patients.

- An aggressive hydration should be avoided and patients should be monitored for signs and symptoms of fluid overload, including weight gain. In the majority of patients, $250 \mathrm{~mL}$ hydration before $\mathrm{CFZ}$ infusion in cycle 1 is sufficient to prevent or reduce any acute renal function impairment. Any additional hydration is based on physician discretion according to the risk of lysis syndrome.

- In case of severe dyspnoea, CFZ should be temporarily discontinued until symptoms disappear or return to baseline levels. Dyspnoea may be caused by fluid overload rather than drug toxicity. If aggressive hydration is not expected to be tolerated by the patient, serum creatinine may be monitored and, if stable, hydration may be decreased or discontinued. Most patients with dyspnoea as primary manifestation of a potential cardiac disease do not typically show an EF impairment or other evidence of myocardial dysfunction. In these patients, CFZ could be restarted as soon as symptoms improve.

- Home blood pressure monitoring is recommended during treatment: if home blood pressure values exceed $>135 / 85 \mathrm{mmHg}$ in at least two measurements, CFZ should be temporarily held and hypertensive therapy should be adjusted until blood pressure target levels are reached $(\leq 135 / 85 \mathrm{mmHg}$; Fig. 2, Table S3). No clear recommendation for an antihypertensive agent can be made in this context, due to the lack of controlled studies. The most commonly prescribed antihypertensive agents are ACEi and ARBs, dihydropyridine calcium channel blockers, ß-blockers and diuretics [34].

- In patients with clinical signs or symptoms suggestive for grade $\geq 2$ cardiac dysfunction, CFZ should be temporarily discontinued until recovery and the following strategy is recommended:

- ECG and echocardiogram with echocardiography-derived strain for diagnostic workup;

- Cardiac magnetic resonance (preferred) or MUGA if echocardiogram is not available or technically feasible (e.g. poor image quality).

- Serum cardiac biomarkers (cardiac troponin, brain natriuretic peptides).

- Cardiologist's referral depending on findings.

No recommendations can be made regarding further continuation or discontinuation of MM-therapy in patients with evidence of cardiac dysfunction during treatment, as long as cardiac function has recovered to grade 1 or baseline. This decision should be taken by the haematologist in close collaboration with the cardiologist, evaluating both the clinical circumstances and the risks/ benefits of continuation of therapy responsible for the cardiac dysfunction.

Carfilzomib relationship with the emerging CVAE should be assessed. If grade 3/4 CVAEs are related to $\mathrm{CFZ}$ use, dose reductions or definitive discontinuation may be needed.

Carfilzomib treatment could be restarted at the dose used before the event or at a reduced dose if the CVAE was not related to CFZ. 
Specific thromboprophylaxis strategies are recommended in MM patients regardless of $\mathrm{CFZ}$ treatment and have been discussed elsewhere [35].

\section{Conclusions}

With the use of cardiotoxic drugs, haematologists need to develop strategies to identify and manage cardiovascular risk in clinical investigations and in general practice. The highly effective agent $\mathrm{CFZ}$ is associated with CVAE risks. Since this agent has shown to improve both PFS and OS compared to standard treatment in RRMM patients, avoiding toxicities that may prevent patient access to $\mathrm{CFZ}$ has become a priority. However, the risk-benefit ratio for an agent should be interpreted depending on the nature and severity of the disease, and restrictive approaches can potentially delay or prevent the access to innovative treatments. This consensus paper considers the best available present evidence and the application of data from large trials and provides clinically useful recommendations and treatment algorithms for its safe use (Figs 1 and 2). Future studies should prospectively analyse the mechanism of cardiovascular damage, the risk factors of developing CVAEs (including new techniques such as global longitudinal strain) and the potential role of cardio-protective drugs.

\section{Conflict of interest statement}

Sara Bringhen has received honoraria from Bristol-Myers Squibb, Celgene, Amgen and Janssen; has served on the advisory boards for Amgen and Janssen; and has undertaken consultancy for Takeda. Alberto Milan has received speaker and/or consulting honoraria from Amgen and Boehringer. Francesca Gay has received honoraria from Takeda, Amgen, Celgene, Janssen, and Bristol-Myers Squibb; has served on the advisory boards for Amgen, Bristol-Myers Squibb, Celgene, Janssen, Roche, and Takeda. Alessandra Larocca has received honoraria from Amgen, BristolMyers Squibb, Celgene, and Janssen; has served on the advisory boards for Bristol-Myers Squibb, Celgene, Janssen, and Takeda. Massimo Offidani has received honoraria from and served on the advisory boards for Celgene, Janssen, Takeda, Amgen, and Bristol-Myers Squibb. Evangelos Terpos has received honoraria from Amgen, Celgene, Genesis, Janssen, Novartis, Takeda, AbbVie, Bristol-Myers Squibb, and GSK; research funding from Celgene, Janssen, and Amgen; has participated in DMC for Celgene and in SC for
Amgen, Takeda and Janssen. Hartmut Goldschmidt has received research support (Institutions) from Amgen, Bristol-Myers Squibb, Celgene, Chugai, Janssen, Sanof, Mundipharma, Takeda, and Novartis; honoraria (Speakers' Bureaus) from Celgene, Janssen, Novartis, Chugai, Bristol-Myers Squibb and ArtTempi; has served on the advisory boards (Institutions) for Adaptive Biotechnology, Amgen, Bristol-Myers Squibb, Celgene, Janssen, Sanofi, and Takeda. Michele Cavo has received honoraria from Janssen, Amgen, Bristol-Myers Squibb, and Celgene. Holger W. Auner has received research support from Amgen; has served on the advisory boards for and received honoraria from Amgen, Takeda, Karyopharm, Chugai, and Novartis. Jo Caers has served on the advisory boards for and received honoraria from Amgen, Celgene, and Janssen; has received research funding from Celgene. Meletios A. Dimopoulos has received honoraria from consultancy and advisory boards from Celgene, Janssen, Amgen, Takeda, and BristolMyers Squibb. Mario Boccadoro has received honoraria from Sanofi, Celgene, Amgen, Janssen, Novartis, AbbVie, and Bristol-Myers Squibb; has received research funding from Celgene, Janssen, Amgen, Bristol-Myers Squibb, Mundipharma, Novartis, and Sanofi. Hermann Einsele has served on the advisory boards for, and received honoraria and research support from Janssen and Celgene, and he is a member of their speaker's bureaus; he has received honoraria from Amgen and Novartis and he is a member of their speaker's bureaus. Pieter Sonneveld has served on the advisory boards for and received honoraria from Amgen, Celgene, Janssen, Karyopharm and Takeda-Millennium; has received research support from Amgen, Celgene, Janssen, Takeda-Millennium, and SkylineDx. Monika Engelhardt has received honoraria and research support from Bristol-Myers Squibb, Celgene, Amgen and Janssen; has served on the advisory boards for Amgen and Janssen. The remaining authors have no relevant conflicts of interest.

\section{Funding}

No funding was provided for this guideline article.

\section{Acknowledgements}

Holger W. Auner acknowledges the support of the Imperial College London National Institute of Health Research-Biomedical Research Centre 
(NIHR-BRC) and the Imperial College London Cancer Research UK Centre.

\section{Author contributions}

Concept and design: Bringhen, Milan, D'Agostino, Ferri, Boccadoro, Einsele, Sonneveld, Engelhardt. Acquisition, analysis, or interpretation of data: all authors. Drafting of the manuscript: Bringhen, Milan, D'Agostino, Boccadoro, Einsele, Sonneveld, Engelhardt. Statistical analysis: Bringhen, Milan, D'Agostino. Administrative, technical or material support: Bringhen, Milan, D’Agostino. Supervision: Bringhen, Milan, Boccadoro, Einsele, Sonneveld, Engelhardt. Critical revision of the manuscript for important intellectual content: all authors. Final approval of the version to be published: all authors. Agreement to be accountable for all aspects of the work in ensuring that questions related to the accuracy or integrity of any part of the work are appropriately investigated and resolved: all authors.

\section{References}

1 Stewart AK, Rajkumar SV, Dimopoulos MA et al. Carfilzomib, lenalidomide, and dexamethasone for relapsed multiple myeloma. $N$ Engl J Med 2015; 372: 142-52.

2 Dimopoulos MA, Moreau P, Palumbo A et al. Carfilzomib and dexamethasone versus bortezomib and dexamethasone for patients with relapsed or refractory multiple myeloma (ENDEAVOR): a randomised, phase 3, open-label, multicentre study. Lancet Oncol 2016; 17: 27-38.

3 Siegel DS, Oriol A, Rajnics P et al. Updated results from ASPIRE and ENDEAVOR, randomized, open-label, multicenter phase 3 studies of carfilzomib in patients (Pts) with relapsed/refractory multiple myeloma (RRMM). Clin Lymphoma Myeloma Leuk 2017; 17: e142 [Abstract \#PS-254 (d), IMW 2017].

4 Waxman AJ, Clasen S, Hwang W-T et al. Carfilzomibassociated cardiovascular adverse events. JAMA Oncol 2018; 4: e174519.

5 Zamorano JL, Lancellotti P, Rodriguez Muñoz D et al. 2016 ESC Position Paper on cancer treatments and cardiovascular toxicity developed under the auspices of the ESC Committee for Practice Guidelines: the Task Force for cancer treatments and cardiovascular toxicity of the European Society of Cardiology (ESC). Eur Heart $J$ 2016; 37: 2768-801.

6 Siegel D, Martin T, Nooka A et al. Integrated safety profile of single-agent carfilzomib: experience from 526 patients enrolled in 4 phase II clinical studies. Haematologica 2013; 98: $1753-61$.

7 Hájek R, Russell SD, Lyon A et al. A substudy of the phase 3 ENDEAVOR study: serial echocardiographic assessment of patients with relapsed multiple myeloma (RMM) receiving carfilzomib plus dexamethasone or bortezomib plus dexamethasone. Haematologica 2016; 101(s1): 263. [Abstract \#P664, EHA 2016 21st Congress].
8 Hájek R, Masszi T, Petrucci MT et al. A randomized phase III study of carfilzomib vs low-dose corticosteroids with optional cyclophosphamide in relapsed and refractory multiple myeloma (FOCUS). Leukemia 2017; 31: 107-14.

9 Berenson JR, Cartmell A, Bessudo A et al. CHAMPION-1: a phase $1 / 2$ study of once-weekly carfilzomib and dexamethasone for relapsed or refractory multiple myeloma. Blood 2016; 127: $3360-8$.

10 Delforge M, Ludwig H. How I manage the toxicities of myeloma drugs. Blood 2017; 129: 2359-67.

11 Ludwig H, Delforge M, Facon T et al. Prevention and management of adverse events of novel agents in multiple myeloma: a consensus of the European Myeloma Network. Leukemia 2018; 32: 1542-60.

12 Dimopoulos MA, Roussou M, Gavriatopoulou M et al. Cardiac and renal complications of carfilzomib in patients with multiple myeloma. Blood Adv 2017; 1: 449-54.

13 Tannous $\mathrm{P}$, Zhu $\mathrm{H}$, Nemchenko A et al. Intracellular protein aggregation is a proximal trigger of cardiomyocyte autophagy. Circulation 2008; 117: 3070-8.

14 Efentakis P, Kremastiotis G, Varela A et al. Molecular mechanisms of carfilzomib-induced cardiotoxicity in mice and the emerging cardioprotective role of metformin. Blood 2019; 133: $710-23$.

15 Brandes RP. Endothelial dysfunction and hypertension. Hypertens 2014; 64: 924-8.

16 Mancia G, Fagard R, Narkiewicz K et al. 2013 ESH/ESC guidelines for the management of arterial hypertension: the Task Force for the Management of Arterial Hypertension of the European Society of Hypertension (ESH) and of the European Society of Cardiology (ESC). Eur Heart $J$ 2013; 34: 2159-219.

17 Conroy R, Pyörälä K, Fitzgerald AP et al. Estimation of tenyear risk of fatal cardiovascular disease in Europe: the SCORE project. Eur Heart J 2003; 24: 987-1003.

18 Hodgkinson J, Mant J, Martin U et al. Relative effectiveness of clinic and home blood pressure monitoring compared with ambulatory blood pressure monitoring in diagnosis of hypertension: systematic review. BMJ 2011; 342: d 3621 .

19 Marwick TH, Gillebert TC, Aurigemma G et al. Recommendations on the use of echocardiography in adult hypertension: a report from the European Association of Cardiovascular Imaging (EACVI) and the American Society of Echocardiography (ASE). Eur Hear J - Cardiovasc Imaging 2015; 16: 577605.

20 Zito C, Longobardo L, Cadeddu C et al. Cardiovascular imaging in the diagnosis and monitoring of cardiotoxicity. $J$ Cardiovasc Med 2016; 17: e35-44.

21 Lang RM, Badano LP, Mor-Avi V et al. Recommendations for cardiac chamber quantification by echocardiography in adults: an update from the American Society of Echocardiography and the European Association of Cardiovascular Imaging. $J$ Am Soc Echocardiogr 2015; 28: 139 e 14.

22 Mor-Avi V, Lang RM, Badano LP et al. Current and evolving echocardiographic techniques for the quantitative evaluation of cardiac mechanics: ASE/EAE consensus statement on methodology and indications endorsed by the Japanese Society of Echocardiography. Eur J Echocardiogr 2011; 12: 167-205. 
23 Mele D, Rizzo P, Pollina AV, Fiorencis A, Ferrari R. Cancer therapy-induced cardiotoxicity: role of ultrasound deformation imaging as an aid to early diagnosis. Ultrasound Med Biol 2015; 41: 627-43.

24 Krishnasamy R, Isbel NM, Hawley CM et al. Left ventricular global longitudinal strain (GLS) is a superior predictor of allcause and cardiovascular mortality when compared to ejection fraction in advanced chronic kidney disease. Reboldi G, ed. PLoS ONE 2015; 10: e0127044.

25 Christenson ES, James T, Agrawal V, Park BH. Use of biomarkers for the assessment of chemotherapy-induced cardiac toxicity. Clin Biochem 2015; 48: 223-35.

26 Plana JC, Galderisi M, Barac A et al. Expert consensus for multimodality imaging evaluation of adult patients during and after cancer therapy: a report from the American Society of Echocardiography and the European Association of Cardiovascular Imaging. J Am Soc Echocardiogr 2014; 27: 911-39.

27 Iannaccone A, Bruno G, Ravera A et al. Evaluation of cardiovascular toxicity associated with treatments containing proteasome inhibitors in multiple myeloma therapy. High Blood Press Cardiovasc Prev 2018; 25: 209-18.

28 Neilan TG, Coelho-Filho OR, Pena-Herrera D et al. Left ventricular mass in patients with a cardiomyopathy after treatment with anthracyclines. Am J Cardiol 2012; 110: 1679-86.

29 Gottdiener JS, Mathisen DJ, Borer JS et al. Doxorubicin cardiotoxicity: assessment of late left ventricular dysfunction by radionuclide cineangiography. Ann Intern Med 1981; 94: 430-5.

30 Cadeddu C, Mercurio V, Spallarossa P et al. Preventing antiblastic drug-related cardiomyopathy. $J$ Cardiovasc Med 2016; 17: S64-75.

31 Boucek RJ, Steele A, Miracle A, Atkinson J. Effects of angiotensin-converting enzyme inhibitor on delayed-onset doxorubicin-induced cardiotoxicity. Cardiovasc Toxicol 2003; 3: 319-30.

32 Bosch X, Rovira M, Sitges M et al. Enalapril and carvedilol for preventing chemotherapy-induced left ventricular systolic dysfunction in patients with malignant hemopathies: the OVERCOME Trial (preventiOn of left Ventricular dysfunction with Enalapril and caRvedilol in patients submitted to intensive Chemotherapy for the treatment of Malignant hemopathies). J Am Coll Cardiol 2013; 61: 2355-62.

33 Gulati G, Heck SL, Ree $\mathrm{AH}$ et al. Prevention of cardiac dysfunction during adjuvant breast cancer therapy (PRADA): a $2 \times 2$ factorial, randomized, placebo-controlled, doubleblind clinical trial of candesartan and metoprolol. Eur Heart $J$ 2016; 37: 1671-80.
34 Milan A, Puglisi E, Ferrari L, Bruno G, Losano I, Veglio F. Arterial hypertension and cancer. Int $J$ Cancer 2014; 134: 2269-77.

35 Palumbo A, Rajkumar SV, Dimopoulos MA et al. Prevention of thalidomide- and lenalidomide-associated thrombosis in myeloma. Leukemia 2008; 22: 414-23.

36 Williams B, Mancia G, Spiering W et al. 2018 ESC/ESH Guidelines for the management of arterial hypertension. Eur Heart J 2018; 39: 3021-104.

Correspondence: Sara Bringhen, MD, PhD, Myeloma Unit, Division of Hematology, University of Torino, Azienda OspedalieroUniversitaria Città della Salute e della Scienza di Torino, via Genova 3, 10122 - Torino, Italy.

(fax: 00390116334187 ; e-mail: sarabringhen@yahoo.com).

\section{Supporting Information}

Additional Supporting Information may be found in the online version of this article:

Data S1. Currently available guidelines on cancer treatments and cardiotoxicity.

Data S2. Role of biomarkers.

Data S3. Role of nutritional supplementation and exercise training on prevention of chemotherapyinduced cardiotoxicity in cancer patient.

Table S1. Patients who are at increased risk for cardiac dysfunction according to the American Society of Clinical Oncology Clinical Practice Guidelines.

Table S2. Baseline risk factors for cardiotoxicity.

Table s3. Office blood pressure treatment target range.

Figure S1. Target organ damage in hypertension 